\title{
Surface-induced low-field instability of antiferromagnetic multilayers
}

\author{
A. L. Dantas and A. S. Carriço* \\ Departamento de Física Teórica e Experimental, Universidade Federal do Rio Grande do Norte, CEP 59.072-970-Natal, RN, Brazil
}

(Received 3 August 1998)

\begin{abstract}
We discuss the surface-induced low-field instability of the antiferromagnetic phase of magnetic multilayers. The threshold field is calculated analytically for multilayers of arbitrary thickness containing an even number of layers. We show that the threshold is given by $H_{S S F}=\sqrt{H_{e} H_{a}+H_{a}^{2}}$, where $H_{e}$ and $H_{a}$ are the effective exchange and anisotropy fields. The effective anisotropy field $H_{a}$ may include both uniaxial and fourfold crystalline anisotropy. Numerical simulations of the equilibrium phases, based on a self-consistent effective field method, are used to obtain the magnetization pattern. We find that thick uniaxial multilayers display a three-stage transition from the antiferromagnetic to the field-aligned phases, whereas in thin multilayers the transition is from the antiferromagnetic to a nearly spin-flop structure, which gradually aligns with the applied field. If the films composing the multilayer have uniaxial and crystalline anisotropy, the magnetization profile in the multilayer and the nature of the transition depend on the relative values of the uniaxial and crystalline anisotropies. [S0163-1829(99)11301-8]
\end{abstract}

\section{INTRODUCTION}

The properties of magnetic multilayers have recently attracted a great deal of research interest. This is largely due to the technological potential of the measured giant magnetoresistance $^{1}$ of transition metal multilayers. Also the possibility of tailoring a wide class of magnetic multilayers, with films down to a few atomic planes thicknesses, has motivated basic research. New phases not encountered in the parent materials have been found. The magnetic properties of these structures depend significantly on the layering pattern and have been the subject of a great deal of experimental and theoretical work in the last decade. ${ }^{2}$

A large fraction of phenomena in magnetic multilayers has been so far studied by assuming invariance in the directions parallel to the interfaces, since the main features induced by surfaces originate in the magnetization variations in the direction perpendicular to the surface. Therefore these systems have most commonly been modeled by a stacking of inequivalent moments, each representing a layer parallel to the surface, coupled through an effective exchange field and subjected to anisotropy fields. This covers aspects of major interest, like surface and interface effects, size effects, and mutual stabilization between components of magnetic superlattices. ${ }^{3}$

Surface effects are particularly relevant to the stability of the antiferromagnetic phase of antiferromagnetic (AF) multilayers. Surface spins are softer and can more easily be turned in the direction of the applied field. In fact a surfacenucleated field-induced phase transition has been predicted for two-sublattice uniaxial antiferromagnets ${ }^{4}$ and shown to occur at a lower field strength than the bulk spin-flop field. In this work the equilibrium equations were solved for a semiinfinite system of spins coupled antiferromagnetically, through an exchange field $H_{e}$, and subjected to an external field along the easy axis of the uniaxial anisotropy. It was shown that the phase transition occurs when an external field of magnitude given by $H_{\text {ext }}=\sqrt{H_{e} H_{a}}$ is applied antiparallel to surface spins. In this work the limit of $H_{a} \ll H_{e}$ was ex- plored and terms proportional to the anisotropy field were neglected. In the bulk the AF phase becomes unstable at an applied field strength of $H_{S F}=\sqrt{2 H_{e} H_{a}+H_{a}^{2}} . H_{e}$ and $H_{a}$ are the exchange and anisotropy fields and $H_{S F}$ is the bulk spinflop field. Therefore the surface-mediated instability occurs at a field much lower than the bulk spin-flop field for lowanisotropy antiferromagnetic materials.

Recently it has been shown that a low-field surfaceinduced spin-flop transition also occurs in uniaxial antiferromagnetic films. ${ }^{5}$ These results were for two-sublattice uniaxial antiferromagnetic fluorides $\left(\mathrm{FeF}_{2}, \mathrm{CoF}_{2}, \mathrm{MnF}_{2}\right)$. At a field strength lower than the bulk spin-flop field a phase transition is nucleated at the surface where spins point opposite to the applied field. The same threshold field was found for films containing an even number of planes, no matter how large is the number of planes.

For antiferromagnetic films with an odd number of layers size effects are relevant. In this case the surface spins are softer and more easily kept parallel to the applied field. Therefore the threshold field for AF instability is larger than the bulk value. We have shown ${ }^{5}$ that the threshold field for the surface-induced spin-flop transition is thickness dependent. For thin films the transition requires large applied fields. For thick films the threshold field was shown to be equal to the bulk spin-flop field.

To our knowledge, there is no experimental report on the surface-induced instability of antiferromagnets. However, a stacking of thin uniaxial ferromagnetic films coupled through nonmagnetic spacers can be regarded isomorphic to a twosublattice antiferromagnet, if the interfilm coupling favors an antiferromagnetic alignment of neighboring films. The first experimental verification of the surface spin-flop transition was reported on transition metal multilayers. The simultaneous analysis of Magneto-optic Kerr effect (MOKE) and superconducting quantum interference device (SQUID) magnetization curves of a $\mathrm{Fe} / \mathrm{Cr}$ multilayer demonstrated clearly that the transition is nucleated at the surface where spins point opposite to the applied field. ${ }^{6}$

Brillouin light scattering as well as ferromagnetic reso- 
nance (FMR) provide useful information regarding the exchange and anisotropy fields. ${ }^{7}$ Typical values of the exchange and anisotropy fields in transition metal multilayers are found in recent experimental data ${ }^{8-13}$ and review articles. ${ }^{14}$ In a number of metallic multilayers of current interest the magnetic symmetry is controlled by a combination of crystalline anisotropy and surface- (or strain-) induced uniaxial anisotropy. The relative strength of these contributions to the effective anisotropy of thin films depends on a number of factors, including the growth process itself, the substrate, and the crystallographic orientation of the stacking of films. The surface contribution typically varies as the inverse of the magnetic film thickness, leading in some cases to a crossover, at thicknesses of the order of a few angstroms, between the uniaxial-anisotropy- and the crystallineanisotropy-dominated regimes.

The orientation of the surface-induced anisotropy easy axis with respect to the crystalline anisotropy easy directions is of particular interest. In a recent report the magnetic properties of $\mathrm{Fe} / \mathrm{Cr}(211)$ superlattices grown on $\mathrm{MgO}(110)$ substrates were studied. ${ }^{11}$ It was shown that Fe films exhibit a strong uniaxial anisotropy along the $[0, \overline{1}, 1]$ direction in the $\mathrm{Fe}(211)$ plane, with the easy axis making an angle of approximately $40^{\circ}$ with the easy axis of the crystalline anisotropy. Therefore the uniaxial axis is nearly in the hard direction of the crystalline anisotropy. In this work the authors found an appreciable change in the uniaxial anisotropy if the Fe thickness is varied from 14 to $90 \AA$, while the reported values of the crystalline anisotropy are practically equal to the bulk value. The crossover inverse thickness, for which the uniaxial and crystalline anisotropy energies are equal, is around $0.04 \AA^{-1}$. This corresponds to an Fe film thickness of $t_{\mathrm{Fe}}=25 \AA$ (see Fig. 3 of Ref. 11).

The field dependence of the giant magnetoresistance of transition metal antiferromagnetic multilayers is associated with the changes in the relative orientation of the magnetizations of the ferromagnetic layers. ${ }^{1}$ Therefore the threshold field for instability of the antiferromagnetic phase of these multilayers is a key parameter. The effective exchange of transition metal multilayers varies with spacer thickness. Therefore the value of the anisotropy energy is not necessarily small compared to the effective exchange energy. The anisotropy to exchange ratio may vary significantly according to the stacking pattern. Thus, it is useful to obtain a general expression for the threshold field, valid for any values of the exchange and anisotropy fields.

We presently investigate the low-field surface-induced instability of the AF phase of an antiferromagnetic multilayer. The results apply to two-sublattice uniaxial antiferromagnetic thin films as well as to antiferromagnetic multilayers constructed with thin transition metal films. We show that, for AF multilayers with an even number of ferromagnetic layers, the threshold field for instability of the AF phase is not dependent on the multilayer thickness. Therefore it provides one more function of the exchange and anisotropy fields for the interpretation of the magnetic properties of antiferromagnetic multilayers.

Furthermore, the large variation of magnetization in the field-induced instability of the AF state might be of practical relevance. Antiferromagnetic multilayers with an odd number of ferromagnetic films exhibit finite magnetization at zero applied field. However, if the number of ferromagnetic films is even, then the net magnetization of the multilayer is zero. We show presently that the magnetization jump at the field-induced transition is controlled by the nature of the anisotropy of the ferromagnetic films composing the multilayer.

We restrict our present analysis to multilayers with an even number of layers. For these multilayers, in the antiferromagnetic state, the spins of one of the surfaces are opposite to the spins of the other surface of the multilayer. Therefore one of the surfaces of the multilayer has spins opposite to the external field. The instability of the antiferromagnetic state is nucleated at this surface. This is a genuine surface effect and requires a lower value of the external field strength, compared to the bulk spin-flop field. Therefore it may have interest for the study of multilayers designed for devices that should respond at low field values. Furthermore, the value of the external field strength which produces the instability is independent of the multilayer thickness. ${ }^{5}$ Thus, it is possible to calculate analytically the threshold field as a function of the characteristic fields (exchange and anisotropy) of the multilayer.

If the multilayer contains an odd number of layers, then the nature of the process that leads to the instability of the antiferromagnetic state is distinct. In this case it is not possible to single out a particular layer where the nucleation of the instability occurs. Instead, the multilayer as a whole responds to the external field, and the value of the threshold field is dependent upon the surface to volume ratio of the multilayer. For a multilayer with $N$ magnetic layers, the surface to volume ratio is $2 /(N-2)$. For large values of $N$ the contribution of the surface region to the magnetic energy is negligible. In this limit the threshold field is equal to the bulk spin-flop field. However, for small values of $N$ surface effects are relevant. The surface spins, with lower coordination, are more easily kept parallel to the external field. Thus the effect of surfaces in odd-numbered thin multilayers is to increase the threshold field for instability of the antiferromagnetic state. We have found that in this case the threshold field for instability of the antiferromagnetic state may be much larger than the bulk spin-flop field. ${ }^{5}$ We have shown that as the surface to volume ratio is decreased, the threshold field drops and reaches the value of the bulk spin-flop field for sufficiently thick multilayers. Therefore thin multilayers have large values of the threshold field. This case is less attractive for current applications, since modern devices based on magnetic multilayers are designed to respond at low values of the external field strength. Furthermore, it is not practical to calculate analytically the threshold field, since it depends on the multilayer thickness. Thus, we presently concentrate on a study of multilayers with an even number of layers.

The basic structure of the calculation is initially set for an antiferromagnetically coupled stacking of ferromagnetic layers with uniaxial anisotropy in Sec. II. In Sec. III the theory is extended to include a contribution from fourfold symmetry crystalline anisotropy. In the last two sections we discuss the nature of the transition according to the relative strengths of the uniaxial and crystalline anisotropies and present our conclusions. 


\section{FIELD-INDUCED INSTABILITY}

We consider each layer of the multilayer represented by a single spin variable $\vec{S}_{n}$, with components only in the plane of the layer. We consider only nearest neighbor interactions and call $z$ the uniaxial axis and $y$ the axis normal to the surface. The external field is along the uniaxial axis and only multilayers with an even number of layers $(N)$ are considered.

We use the principle of induction to examine the stability of the antiferromagnetic configuration. First a system consisting of a pair of layers is considered. Then a multilayer with four layers is studied and finally we show that the threshold field for a multilayer with $N+2$ layers is the same as that of a multilayer with $N$ layers. Thus, the threshold field is an intrinsic value. It is defined by the exchange and anisotropy fields of the multilayer, and is not dependent upon the number of layers, $N$.

The internal energy, written in units of $g \mu_{B} H_{E} S$, where $H_{E}$ is the exchange field coupling neighboring layers, is given by

$$
E=\sum_{n=1}^{N}\left\{\frac{1}{2} \cos \left(\theta_{n}-\theta_{n+1}\right)-\frac{\alpha}{2} \cos ^{2} \theta_{n}-h \cos \theta_{n}\right\} .
$$

The first term corresponds to the exchange coupling between adjacent layers, the second is the uniaxial anisotropy energy, and the third term is the Zeeman energy. $\alpha=H_{a} / H_{e}$ and $h$ $=H / H_{e}$ are the uniaxial anisotropy field, and applied field, in units of exchange field. The exchange term is not included for the $N$ th layer.

The magnetic phases are described by the angles $\left\{\theta_{i}, i\right.$ $=1, \ldots, N\}$. A given profile corresponds to an extremum of the energy if $\partial E / \partial \theta_{i}=0$ for $i=1,2, \ldots, N$. The relevant equations are

$$
\begin{gathered}
\frac{\partial E}{\partial \theta_{1}}=\left(h+\alpha \cos \theta_{1}\right) \sin \theta_{1}-\frac{1}{2} \sin \left(\theta_{1}-\theta_{2}\right)=0, \\
\frac{\partial E}{\partial \theta_{2}}=\left(h+\alpha \cos \theta_{2}\right) \sin \theta_{2}-\frac{1}{2} \sin \left(\theta_{2}-\theta_{1}\right) \\
\quad+\sin \left(\theta_{2}-\theta_{3}\right)=0, \\
\frac{\partial E}{\partial \theta_{3}}=\left(h+\alpha \cos \theta_{3}\right) \sin \theta_{3}-\frac{1}{2} \sin \left(\theta_{3}-\theta_{2}\right) \\
\quad+\sin \left(\theta_{3}-\theta_{4}\right)=0, \\
\vdots \\
\frac{\partial E}{\partial \theta_{N}}=\left(h+\alpha \cos \theta_{N}\right) \sin \theta_{N}-\frac{1}{2} \sin \left(\theta_{N}-\theta_{N-1}\right)=0 .
\end{gathered}
$$

The $N$ conditions imposed by Eqs. (2) are automatically satisfied in the AF phase, where the angles $\left(\theta_{i}\right)$ alternate between $\pi$ and 0 . However, in order to satisfy the condition for a minimum of the energy it is necessary that all the eigenvalues of the matrix $M$, formed with elements given by $m_{i j}=\partial^{2} E / \partial \theta_{i} \partial \theta_{j}$, be positive. ${ }^{15}$ The matrix elements $m_{i j}$ are given by

$$
\begin{gathered}
m_{i i}=\left(h+\alpha \cos \theta_{i}\right) \cos \theta_{i}-\frac{\alpha}{2} \sin 2 \theta_{i}-\frac{1}{2}\left[\cos \left(\theta_{i-} \theta_{i-1}\right)\right. \\
\left.+\cos \left(\theta_{i-} \theta_{i+1}\right)\right], \\
m_{i j}=\frac{1}{2} \cos \left(\theta_{i}-\theta_{j}\right)\left(\delta_{j, i+1}+\delta_{j, i-1}\right),
\end{gathered}
$$

where $\delta_{n m}$ is the Kronecker delta function.

In the AF phase the matrix elements $m_{i j}$ are constructed from Eqs. (3), with $\theta_{i}-\theta_{i+1}=\pi$. For surface spins the exchange part in the diagonal elements, $m_{i i}$, contains only one of the cosine terms. If the applied field is smaller than the threshold for instability of the AF phase, all the eigenvalues of the matrix $M$ are positive. Instability occurs when one eigenvalue becomes zero. In this case the matrix $M$ becomes singular. The elements of the matrix $M$ depend on the value of the magnetic field, and the critical field for instability of the AF phase is calculated by finding the lowest field value for which the determinant of $M$ is zero.

For a multilayer with $N$ ferromagnetic layers, $M$ is an $N \times N$ matrix and, except for the matrix elements of the principal diagonal and the two secondary diagonals, all the matrix elements are zero. The elements of the principal diagonal alternate between those corresponding to positive and negative values of the Zeeman energy. Also the matrix elements $m_{11}$ and $m_{N N}$ are distinct by having only half coordination and, therefore, half the exchange of the others.

For a pair of layers $M$ is given by

$$
M_{2}=\left(\begin{array}{ll}
a & b \\
b & c
\end{array}\right)
$$

where we have used $a=-h+\alpha+1 / 2, c=h+\alpha+1 / 2$, and $b=-1 / 2$. These matrix elements correspond to choosing $\theta_{1}=\pi$ and $\theta_{2}=0$. The AF instability occurs when $a c=b^{2}$. This corresponds to an applied field $H_{S S F}$ given by

$$
H_{S S F}=\sqrt{H_{e} H_{a}+H_{a}^{2}} \text {. }
$$

Notice that the decrease in the threshold field, compared to the bulk value $\sqrt{2 H_{e} H_{a}+H_{a}^{2}}$, corresponding to an infinite stacking of layers, results from the reduced exchange of both spins.

For a multilayer with four layers we have

$$
M_{4}=\left(\begin{array}{cccc}
a & b & 0 & 0 \\
b & c-b & b & 0 \\
0 & b & a-b & b \\
0 & 0 & b & c
\end{array}\right) .
$$

We use elementary matrix algebra to write the determinant of $M$ as the product of the diagonal elements $d_{n}$ of the upper triangular matrix 


$$
D_{4}=\left(\begin{array}{cccc}
a & b & 0 & 0 \\
0 & c-b-\frac{b^{2}}{a} & b & 0 \\
0 & 0 & a-b-\frac{b^{2}}{c-b-\frac{b^{2}}{a}} & b \\
0 & 0 & 0 & c-\frac{b^{2}}{a-b-\frac{b^{2}}{c-b-\frac{b^{2}}{a}}}
\end{array} \mid .\right.
$$

Therefore

$$
\operatorname{Det}\left(M_{4}\right)=a\left(c-b-\frac{b^{2}}{a}\right)\left(a-b-\frac{b^{2}}{c-b-\frac{b^{2}}{a}}\right)\left(c-\frac{b^{2}}{a-b-\frac{b^{2}}{c-b-\frac{b^{2}}{a}}}\right) .
$$

In this case it easy to show that the lowest field value for which the determinant of $M_{4}$ is zero is the field for which $a c=b^{2}$, as in the case of a pair of layers [Eq. (5)]. By inspection of Eq. (8) we find that the factors in $\operatorname{Det}\left(M_{4}\right)$ are given by $a,-b, a$, and 0 if $a c=b^{2}$. The odd-numbered diagonal elements are given by $-h_{S S F}+\alpha+0.5$ (with $h_{S S F}$ $=H_{S S F} / \mathrm{He}$ ), while the $d_{2}=0.5$ and $d_{4}=0$.

The threshold field for a multilayer with $N$ layers is also found by putting $M_{N}$ into an upper triangular form. The determinant of $M_{N}$ is then simply the product of diagonal elements $\left(d_{n}\right)$ of the transformed (upper triangular) matrix $D_{N}$. We have

$$
\operatorname{Det}\left(M_{N}\right)=d_{1} d_{2} d_{3} \cdots d_{N-1} d_{N}^{*} .
$$

The diagonal elements $\left(d_{n}\right)$ are the following functions of $h$ :

$$
\begin{gathered}
d_{1}=a, \\
d_{n}=\left\{\begin{array}{cl}
c-b-\frac{b^{2}}{d_{n-1}} & \text { for } 2 \leqslant n<N \quad(n \text { even }), \\
a-b-\frac{b^{2}}{d_{n-1}} & \text { for } 3 \leqslant n \leqslant N-1 \quad(n \text { odd }), \\
d_{N}^{*}=c-\frac{b^{2}}{d_{N-1}} .
\end{array}\right.
\end{gathered}
$$

We use $d_{N}^{*}$ for the $N$ th diagonal element of $D_{N}$ for a multilayer with $N$ layers. The first and last elements $d_{1}$ and $d_{N}^{*}$ correspond to surface spins, the first pointing opposite to the field direction and the last aligned along the field, in the AF state. The absence of the $b$ term in the expression for $d_{N}^{*}$ results from the reduced coordination. The expression for $d_{1}$ comes from the same fact.
The recurrence relation between the diagonal elements of the matrix $M$ [Eqs. (10)] is obtained from a composition of two continued fractions. The structure of the equations incorporates the fact that in the $\mathrm{AF}$ phase the spins directions alternate along the field and opposite to it. For the discussion that follows it is convenient to group the diagonal elements $\left[d_{n}(H)\right]$ into two sequences, one corresponding to odd values of $n$ and another corresponding to even values of $n$. Therefore the sequences correspond to spins pointing in the direction of the applied field (even sequence) and opposite to it (odd sequence). The recurrence relations for the even and odd sequences are

$$
d_{n}=\left\{\begin{array}{c}
c-b-\frac{b^{2}}{a-b-\frac{b^{2}}{d_{n-2}}} \text { for } 4 \leqslant n<N \quad(n \text { even }), \\
a-b-\frac{b^{2}}{c-b-\frac{b^{2}}{d_{n-2}}} \text { for } 3 \leqslant n \leqslant N-1 \quad(n \text { odd }), \\
d_{N}^{*}=c-\frac{b^{2}}{a-b-\frac{b^{2}}{d_{N-2}} .}
\end{array}\right.
$$

The elements $d_{1}$ and $d_{2}$ are given by Eqs. (10).

If $h=0$, then $c=a$ and both sequences are described by the same continued fraction. In this case we verified that all $d$ 's are positive and that $d_{1}>d_{2}>d_{3}>\cdots>d_{N-1}>d_{N}^{*}$, with $d_{1}=\alpha+1 / 2$ (see Fig. 1).

We have found that for $h>0$ each element of the even sequence initially increases with field and then decreases to converge to $1 / 2$ (for any value of $n<N$ ) at the threshold field. The elements of the odd sequence (for any value of $n$ ) decrease and converge to $-h+\alpha+1 / 2$ at the critical field 


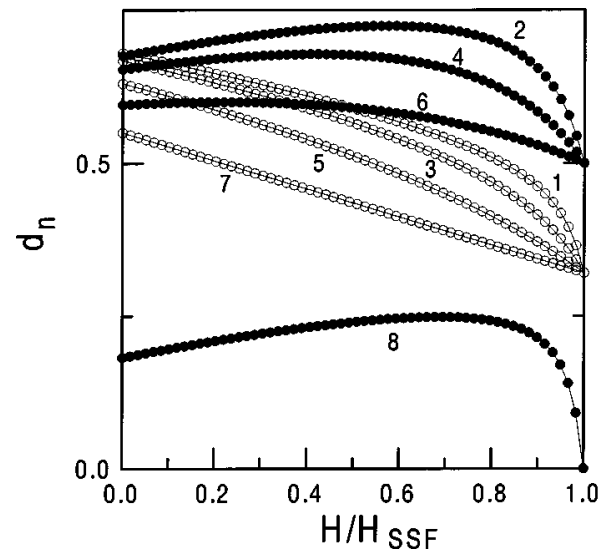

FIG. 1. Field dependence of the diagonal elements $d_{n}$ for a stacking of eight layers. The applied field is shown in units of the surface spin-flop field. The numbers by the curves indicate the values of $n$. Open symbols are used for $n=$ odd and solid symbols are used for $n=$ even. The lines are just a guide to the eyes.

which makes $a c=b^{2}$. Furthermore, $d_{N}^{*}=0$ for $h=h_{S S F}$. Therefore $h_{S S F}$ is the lowest value of the applied field for which the determinant of $M_{N}$ vanishes. This is the threshold field for instability of the $\mathrm{AF}$ state.

At the threshold field $h=h_{S S F}$, the limits of the even and odd sequences can be derived from Eqs. (10) and (11). Starting with $d_{1}=a$ we obtain, from Eqs. (10), $d_{2}=-b$ if $a c$ $=b^{2}$. For the even sequence, from Eqs. (11), for any value of $n<N$, we have $d_{n}=c-b-b^{2} / a$ if $d_{n-2}=-b$. Since $d_{2}$ $=-b$ when $a c=b^{2}$, any element of the even sequence converges to the same value $\left(d_{n}=-b\right)$. It is also clear from Eqs. (11) that, for this value of the applied field and any even value of $N, d_{N}^{*}=0$.

A similar analysis of the limit of any element $\left(d_{n}\right)$ of the odd sequence, as $h$ approaches $h_{S S F}$, can easily be made. All the elements of the odd sequence are finite for $0<h$ $<h_{S S F}$.

We now show, using the principle of induction, that $h_{S S F}$ is independent of $N$. In other words, the threshold for instability of the AF phase is the same for multilayers with an even number of layers. We start by proving that if $d_{N}^{*}$ is zero for a multilayer with $N$ layers, then $d_{N+2}^{*}$ is also zero.

From Eqs. (11) we find that for a multilayer with $N+2$ layers the last element of the diagonal of $M, d_{N+2}^{*}$, is related to the corresponding element of a multilayer with $N$ layers according to

$$
d_{N+2}^{*}=c-\frac{b^{2}}{a-b-\frac{b^{2}}{-b+d_{N}^{*}}} .
$$

By inspection of Eq. (12) we find that if $d_{N}^{*}=0$ at the field value for which $a c=b^{2}$, then $d_{N+2}^{*}$ is also zero at the same applied field value. From Eq. (4) we have that $d_{2}^{*}=c$ $-b^{2} / a$. Thus $d_{2}^{*}=0$ for $a c=b^{2}$. Then it follows by induction that the result is valid for any even value of $N$.

In Fig. 1 we show the field dependence of the two sequences of diagonal elements of $D_{8}$. We selected a multilayer with eight layers and an anisotropy ratio $\alpha$
$=0.05$. The elements of the even sequence $(n<8)$ converge to 0.5 (at the threshold field) while the elements of the odd sequence converge to $-h_{S S F}+\alpha+0.5$. Notice that for any value of $H, d_{8}^{*}$ is smaller then all the other elements. The choice of $\alpha=0.05$ and $N=8$ is a matter of convenience. The basic features displayed in the field dependence of the diagonal elements $d_{n}(n=1,2, \ldots, 8)$, in Fig. 1 , are also found for any other value of $N$ (even) and $\alpha$.

\section{CRYSTALLINE ANISOTROPY}

Metallic multilayered structures comprising alternating ferromagnetic and nonferromagnetic layers are nearly isomorphic to an antiferromagnetic film. There are, however, special features of the metallic multilayers that may lead to significantly different magnetic behavior. In a metallic multilayer the effective antiferromagnetic exchange, coupling neighboring ferromagnetic layers, can be varied by orders of magnitude by choosing the spacer thickness appropriately. Furthermore, the anisotropy of the individual ferromagnetic layers may have a complex structure. Most commonly there are two major contributions to the effective anisotropy: a uniaxial part induced by strains during the film growth or due to surface effects and a crystalline part which is intrinsic to the material. ${ }^{14}$

We consider multilayers with the magnetic moments in the plane of the films. The actual form of the crystalline anisotropy depends on the crystallographic orientation of the stacking. We presently study a particular case when the crystalline anisotropy has fourfold symmetry in the plane of the layers. In this case the crystalline anisotropy contributes to the magnetic energy with a term, for each layer, of the following form:

$$
E_{c}=\frac{1}{2} K_{c} S_{n x}^{2} S_{n z}^{2}
$$

We made this particular choice of the symmetry of the crystalline anisotropy term to allow a simple discussion of relevant features of the magnetic pattern for applied fields just above the threshold field for instability of the antiferromagnetic phase of the multilayer. The results can be extended to other symmetries without much effort.

For the present discussion we assume that the uniaxial anisotropy determines the orientation of the magnetization of the ferromagnetic layers in the absence of applied fields. We also assume that the equilibrium configuration, in the absence of an external field, consists in an antiferromagnetic arrangement of the ferromagnetic layers. Notice that if $K_{c}$ $>0$, then the easy directions of the crystalline anisotropy are $\theta=0, \pm \pi / 2, \pi$, while if $K_{c}<0$, then the easy directions of the crystalline anisotropy are $\theta= \pm \pi / 4, \pm 3 \pi / 4$.

In order to extend the results of Sec. II, we write the crystalline anisotropy per layer as

$$
E_{c}=\frac{K_{c} S^{4}}{2}\left[\cos \left(\theta_{n}\right) \sin \left(\theta_{n}\right)\right]^{2}=\frac{K_{c} S^{4}}{8} \sin \left(2 \theta_{n}\right)^{2} .
$$

The contributions of this new term to the equilibrium equations [Eqs. (2)] as well as the contributions to the matrix $M$ [Eqs. (3)] are easily calculated. We note that 


$$
\frac{\partial^{2} E_{c}}{\partial \theta_{n}^{2}}=K_{c} S^{4} \cos \left(4 \theta_{n}\right) .
$$

Therefore, in the AF phase ( $\theta_{n}=0$ for $n=$ even and $\theta_{n}$ $=\pi$ for $n=$ odd) the above term [Eq. (15)] just adds a constant to the diagonal elements of the second derivative matrix obtained from the exchange, Zeeman, and uniaxial anisotropy energies [Eqs. (3)]. This corresponds to defining an effective anisotropy parameter which includes the anisotropy field amplitudes for the uniaxial and crystalline contributions.

We define $\alpha_{c}=K_{c} S^{2} / 2 J$ where $J$ is the AF exchange coupling, and obtain the threshold field for instability of the AF phase. In units of the exchange field, the critical field is given by $h_{S S F}=\sqrt{\alpha_{e f f}+\alpha_{e f f}^{2}}$, with an effective anisotropy constant defined by $\alpha_{e f f}=\alpha+\alpha_{c}$.

The effect of adding a contribution from the crystalline anisotropy is to introduce a shift in the calculated threshold field given by Eq. (5). The remaining results of Sec. II are not modified. However, compared to the system with only uniaxial anisotropy, the actual spin profile after the threshold field may differ considerably if the crystalline energy is comparable to the uniaxial energy. As we shall discuss below, this aspect is relevant for the interpretation of MOKE magnetization measurements in metallic multilayers.

Our discussion centers on the existence of a given uniaxial anisotropy; then the possible effects of crystalline anisotropy are introduced. This corresponds to the situation that might be found in thin film transition metal multilayers. We consider two distinct cases according to the orientation of the crystalline anisotropy easy axis with respect to the uniaxial axis.

We note that if $K_{c}>0$, then the uniaxial axis is an easy axis of the crystalline anisotropy. In this case the AF state is further stabilized by the crystalline anisotropy and the threshold field for instability increases. If the uniaxial axis is a hard axis of the crystalline anisotropy $\left(K_{c}<0\right)$, the effective anisotropy parameter $\alpha_{e f f}$ decreases and the instability occurs at a lower field (compared to the purely uniaxial case). If the magnitudes of $\alpha$ and $\alpha_{c}$ are comparable, the threshold field may turn out to be weak compared to the exchange field.

We have found that the order of the phase transition, as well as the width of the surface modified region, containing the layers directly affected at the transition, depend on the magnitudes of the anisotropies and the relative orientation of the easy axis. These two aspects are relevant to the interpretation of MOKE magnetization measurements and will be explored in examples in the next section.

\section{SPIN PROFILES AND THE NATURE OF THE TRANSITION}

In this section we discuss the magnetic structure when the external field strength is equal or larger than the surface spin flop field $\left(h_{S S F}\right)$. We divide the discussion into two parts. In the first part we consider purely uniaxial multilayers. In this case the anisotropy of each ferromagnetic layer is uniaxial and for $h=h_{S S F}$ we find a first-order transition with a jump in the magnetization. In the second part we show that if the

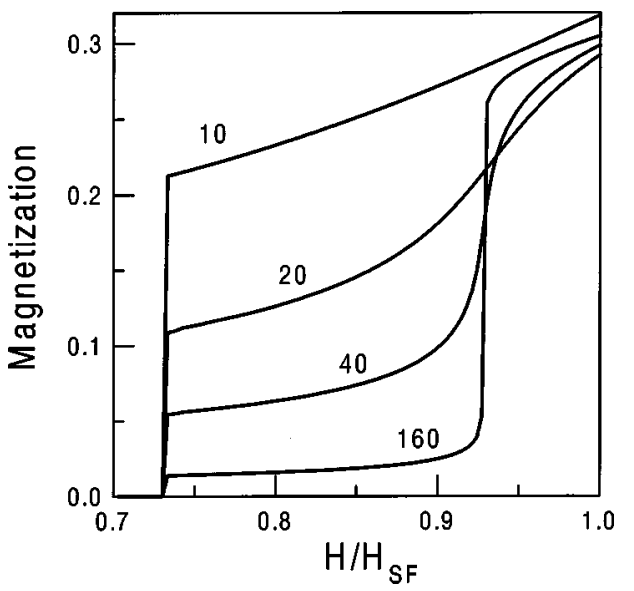

FIG. 2. The magnetization curves for multilayers of various thicknesses and a single value of the uniaxial anisotropy to exchange ratio $(\alpha=0.15)$. The numbers by the curves indicate the number of layers. The applied field is shown in units of the bulk spin-flop field $\left(H_{S F}\right)$, and the magnetization is shown in units of the saturation magnetization.

anisotropy of the ferromagnetic layers is composed of uniaxial and fourfold crystalline anisotropy contributions, then the nature of the phase transition for $h=h_{S S F}$ depends on the orientation of the uniaxial anisotropy field relative to the crystalline anisotropy easy directions.

The spin profiles are calculated for arbitrary field values by a numerical self-consistent algorithm, which consists of finding the equilibrium configuration by allowing the spins to align with the local effective field. This method has been used to study antiferromagnetic films, and the reader is referred to Ref. 3 for details.

For purely uniaxial multilayers we have found that although the critical field is given by Eq. (5) for any value of the anisotropy to exchange fields ratio $\left(\alpha=H_{a} / H_{e}\right)$, the spin profile just after the threshold field is dependent upon the values of $N$ and $\alpha$.

In Fig. 2 we show the magnetization curves for various values of $N$ and a fixed value of $\alpha=0.15$. The magnetization is shown in units of the saturation magnetization, and the applied field is shown in units of the bulk spins flop field $\left(H_{S F}\right)$. For the chosen value of $\alpha$ the surface spin-flop field is given by $H_{S S F}=0.73 H_{S F}$. As seen in Fig. 2 the instability of the antiferromagnetic state occurs at the same value of the applied field $\left(H / H_{S F}=0.73\right)$ for all the multilayers chosen. For the multilayers with $N=10$ and $N=20$, there is a single magnetization jump. For these two cases when $H>H_{S S F}$ the magnetic pattern consists of a surface-modified spin-flop phase (SMSF) in which, except for a few layers near the surfaces, the structure resembles a spin-flop pattern.

For the multilayers with $N>20$ there are two jumps in the magnetization. In these cases for $H=H_{S S F}$ the magnetic phase consists of an almost antiferromagnetic pattern near the surface with spin, in the AF state, parallel to the applied field, and a region with spins canted towards the field direction, near the other surface. When the field is increased, beyond the threshold value, the canted region moves without appreciable change in width to the center of the multilayer. By further increasing the applied field the canted region widens up, initially very slowly until another critical field value 
is reached when the canted region spreads over the whole multilayer, leading to a surface-modified spin-flop phase. In this phase, except for the surface region, the spins are in the spin-flop state. However the angles of orientation with the applied field differ from those for a bulk spin-flop state. These results conform with those reported for $\mathrm{Fe} / \mathrm{Cr}$ multilayers. ${ }^{6}$

The second jump of magnetization in Fig. 2, at $H^{*}$ $\approx 0.92 H_{S F}$, corresponds to the canting of spins largely concentrated in the center of the multilayer spreading over the whole multilayer, leaving the system in a SMSF phase. The major fraction of the spins of thick multilayers is in an almost AF state for lower fields (above the critical value $\left.H_{S S F}\right)$; therefore there is a considerable increase in magnetization in passing to the SMSF state. The threshold field for this transition is size dependent. This is not clearly seen in the picture due to small value of anisotropy $(\alpha=0.15)$ used.

We also note that $H^{*}$ is not the bulk spin-flop transition field. $H^{*}$ is smaller than the bulk spin-flop field. For thick multilayers, when the transition must show unambiguously, the magnetic state before the transition occurs $\left(H_{S S F}<H\right.$ $\left.<H^{*}\right)$ consists of a mixed phase with most of the multilayer in an almost AF state but a fraction of spins in the center of the multilayer is in a canted state similar to a spin-flop phase. The existence of this region of canted spins lowers the stability of the phase with respect to further increase in the applied field, if compared to a purely AF state. Therefore the second transition occurs for a field strength lower then the bulk spin-flop field $\left(H^{*}<H_{S F}\right)$.

We have found that for a given value of $\alpha$ there is a critical number of layers $\left(N_{c}\right)$ below which the above threestage process turns into a two-stage process. For $N<N_{c}$ the surface-modified spin-flop state sets in just above the threshold field. The critical value $N_{c}$ decreases with increasing uniaxial anisotropy.

We now discuss the nature of the field-induced phase transition, for $H=H_{S S F}$, when the anisotropy of the ferromagnetic layers is the sum of two contributions, one with uniaxial symmetry and another with fourfold symmetry. In Fig. 3 we show the total magnetization, for field applied along the uniaxial axis, and profiles of the layer magnetizations for an $\mathrm{AF}$ multilayer with 30 layers. We have chosen $\alpha=0.2$ and $\alpha_{c}= \pm 0.1$. The chosen values of the crystalline anisotropy $\left(\alpha_{c}= \pm 0.1\right)$ are of the same order of magnitude as that of the uniaxial anisotropy. As a result there are relevant differences in the magnetization profile, near the threshold field, if the sign of $\alpha_{c}$ is changed.

In Fig. 3(a) the applied field is shown in units of $H_{S S F}$ for convenience. Notice that $\alpha_{c}=0.1$ corresponds to $\alpha_{e f f}=0.3$ and $\alpha_{c}=-0.1$ corresponds to $\alpha_{e f f}=0.1$. For $\alpha_{c}=0.1$ the transition is of first order and the magnetization jump at the threshold field corresponds to a transition to a surfacemodified spin-flop state which covers all the multilayer. For $\alpha_{c}=-0.1$ the transition is to an almost AF state with weak modifications near the surface with spins opposite to the applied field. In this case the transition is of second order with a continuous increase in the magnetization.

In Fig. 3(b) we display the angles with the $z$ axis at the threshold field. This picture is complementary to Fig. 3(a). The magnetic profiles at the threshold field $\left(H=H_{S S F}\right)$ help to identify the nature of the transition. For $\alpha_{c}=0.1$ the spins
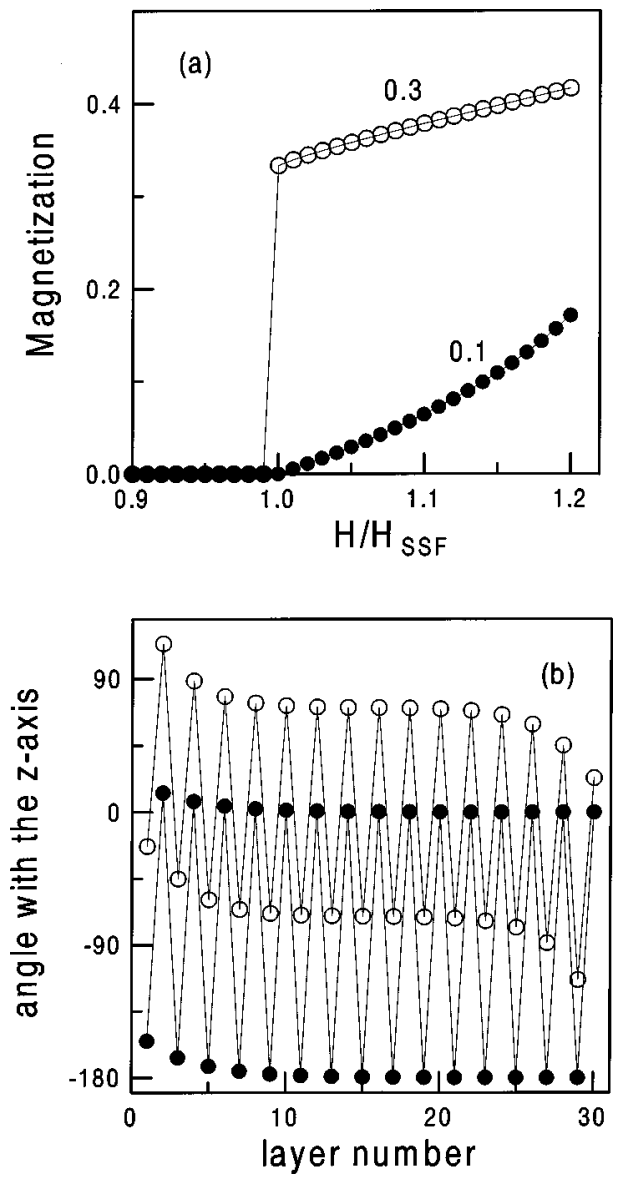

FIG. 3. (a) The magnetization of a 30-layer AF multilayer for positive and negative values of $\alpha_{c}$ (see text for details). The applied field is shown in units of the surface spin-flop field $\left(H_{S S F}\right)$ and the magnetization is shown in units of the saturation magnetization. The uniaxial anisotropy is $\alpha=0.2$ and two values of the effective anisotropy parameter $\alpha_{e f f}$, corresponding to $\alpha_{c}=0.1$ and $\alpha_{c}$ $=-0.1$, are indicated by the numbers by the curves. (b) Profile of the angles $\theta_{n}$, shown in degrees, wih the $z$ axis. Open symbols for $\alpha_{e f f}=0.3$ and solid symbols for $\alpha_{e f f}=0.1$. The lines through the points are just a guide to the eyes.

are almost perpendicular to the applied field. The middle of the multilayer displays a spin-flop pattern and there are small modifications near the surfaces. The magnetic state consists of a SMSF state. The formation of this state is responsible for the jump in the magnetization seen in Fig. 3(a) for $\alpha_{e f f}$ $=0.3$.

For $\alpha_{c}=-0.1$ an almost AF state is seen. The majority of spins is in an AF phase. Only near the surface where spins point opposite to the applied field is there a small field effect. Therefore the total magnetization is rather small, as seen in Fig. 3(a) for $\alpha_{e f f}=0.1$. For this value of $\alpha_{\text {eff }}$ the magnetization increases continuously near the threshold field. Upon further increase in the applied field the almost AF state evolves continuously to a pattern in which a region of canted spins is formed near the surface. This turns into a SMSF state for larger field values.

\section{CONCLUSIONS}

We presented a calculation of the surface-induced lowfield instability of the AF state of a multilayer with negative 
bilinear coupling between the layers and in-plane anisotropies. The results are valid for any value of the effective anisotropy parameter $\left(\alpha_{e f f}\right)$, provided the equilibrium configuration for $H=0$ is an antiferromagnetic arrangement of spins, aligned along the uniaxial easy axis, and for any value of the multilayer thickness, if the number of spins is even.

We started by setting the basic structure of the calculation for uniaxial antiferromagnetic multilayers. These results were extended to include contributions from crystalline anisotropy, so as to apply to the metallic multilayers of current interest.

In the case of a purely uniaxial antiferromagnetic multilayer, the presently calculated threshold field $\left(H_{S S F}\right)$ is the field that makes surface spin waves soft, as shown by Saslow and Mills for a semi-infinite uniaxial antiferromagnet. ${ }^{16}$ We have presently shown that the critical field is thickness independent for finite multilayers, provided there is a surface layer with spins opposite to the applied field.

The first reports on surface-nucleated field-induced phase transitions of AF systems referred to two-sublattice uniaxial antiferromagnets. ${ }^{4}$ These works dealt with the phase transitions of a semi-infinite antiferromagnet. Under the assumption of a small anisotropy to exchange field ratio, the energies of the antiferromagnetic state as well as the energy of the surface-modified spin-flop state were calculated and the threshold field was found as the value of applied field strength which makes these energies equal. Wang et al. reported experimental verification of the surface-induced instability of the AF phase of finite multilayers. ${ }^{6}$ By simultaneous MOKE and SQUID measurements of the magnetization of $\mathrm{Fe} / \mathrm{Cr}$ multilayers with even number of Fe films, it was possible to identify in a clear manner the surface-induced instability. The narrow width of the surface modified region was explored to identify the surface-induced instability.

Our results are consistent with these previous reports. Furthermore, we have shown that $H_{S S F}$ is determined by the values of exchange and anisotropy energies per layer and is independent of the multilayer thickness. In some cases the experimental results indicate that the transition occurs when the applied field strength is such that the energies of the antiferromagnetic and spin-flop-like phases are equal, as pointed out by Wang et al. ${ }^{6}$ The magnetic energy of finite AF multilayers is thickness dependent. Thus it is valuable to have an upper bound for the threshold field. $H_{S S F}$ is a fixed upper bound for the stability of the AF state and should help to examine the magnetic properties of $\mathrm{AF}$ multilayers.
We have also examined the influence of fourfold symmetry crystalline anisotropies. For magnetization in the plane of the layers, we considered a particular kind of symmetry which amounts to adding to the energy of each layer a term of the form $K_{c} S^{2} \cos ^{2}\left(\theta_{n}\right) \sin ^{2}\left(\theta_{n}\right)$. This term affects the energy of the spin-flop-like phase, which forms for applied fields beyond the critical value $H_{S S F}$, and also the actual spin pattern beyond the critical field. However, we have shown that for multilayers containing an even number of ferromagnetic layers the threshold field for instability of the AF state is not thickness dependent. The critical field is given by Eq. (5) with an effective anisotropy parameter $\alpha_{\text {eff }}$ incorporating the uniaxial and crystalline anisotropy energy contributions.

We have shown that either a first-order or a second-order phase transition may occur for a field-induced instability of the AF phase of finite antiferromagnetic multilayers. For multilayers comprising an even number of thin ferromagnetic films with uniaxial anisotropy, the transition is of first order with a jump in the magnetization. In this case the width of the surface-modified region depends both on the multilayer thickness and on the strength of the uniaxial anisotropy field.

If the anisotropy energy of the ferromagnetic films have both uniaxial and fourfold symmetry contributions, then the nature of the phase transition depends on the relative orientation of the easy axis as well as on the relative strength of the anisotropy fields. We have found that if the magnitude of the anisotropy constants ( $\alpha$ and $\alpha_{c}$ ) are comparable, as might be expected for thin films, and the easy axis of the uniaxial anisotropy is along a hard direction of the fourfold anisotropy, then the transition is of second order, with a continuous variation of the magnetization. Furthermore, in this case the critical field may turn out to be rather small, since the critical field is a function of the effective anisotropy parameter $\alpha_{e f f}=\alpha-\alpha_{c}$.

The relevant changes of the giant magnetoresistance of transition metal multilayers set in at a field value which makes the AF state unstable and saturates at a field strength comparable to the exchange field. We hope our present results will be helpful in studying the low-field limit, since $H_{S S F}$ is an upper bound for the stability of the AF state.

\section{ACKNOWLEDGMENT}

This research was partially supported by the CNPq.
*Electronic address: acarrico@dfte.ufrn.br

${ }^{1}$ M. N. Baibich, J. M. Broto, A. Fert, F. Nguyen Van Dau, P. Pretoff, Etiene, G. Creuzet, A. Friedich, and J. Chazelas, Phys. Rev. Lett. 61, 2472 (1988).

${ }^{2}$ R. E. Camley and R. L. Stamps, J. Phys.: Condens. Matter 5, 3727 (1993).

${ }^{3}$ F. C. Nortemann, R. L. Stamps, A. S. Carriço, and R. E. Camley, Phys. Rev. B 46, 10847 (1992); A. S. Carriço and R. E. Camley, ibid. 45, 13117 (1992).

${ }^{4}$ D. L. Mills, Phys. Rev. Lett. 20, 18 (1968); F. Keffer and H. Show, ibid. 31, 1061 (1973).
${ }^{5}$ A. S. Carriço, R. E. Camley, and R. L. Stamps, Phys. Rev. B 50, 13453 (1994).

${ }^{6}$ R. W. Wang, D. L. Mills, E. E. Fullerton, J. E. Mattson, and S. D. Bader, Phys. Rev. Lett. 72, 920 (1994).

${ }^{7}$ P. E. Wigen and Z. Zhang, Braz. J. Phys. 22, 267 (1992).

${ }^{8}$ M. Gester, C. Daboo, R. J. Hicken, S. J. Gray, and J. A. C. Bland, Thin Solid Films 275, 91 (1996).

${ }^{9}$ K. Inomata, Y. Saito, and R. J. Highmore, J. Magn. Magn. Mater. 137, 257 (1994).

${ }^{10}$ P. Kabos, C. E. Patton, M. O. Dima, D. B. Church, R. L. Stamps, and R. E. Camley, J. Appl. Phys. 75, 3553 (1994). 
${ }^{11}$ E. E. Fullerton, M. J. Conover, J. E. Mattson, C. H. Sowers, and S. D. Bader, Phys. Rev. B 48, 15755 (1993).

${ }^{12}$ Y. Roussigné, F. Ganot, C. Dugautier, P. Moch, and D. Renard, Phys. Rev. B 52, 350 (1995).

${ }^{13}$ K. Inomata and Y. Saito, Appl. Phys. Lett. 61, 726 (1992).
${ }^{14}$ See, for instance, B. Heinrich and J. F. Cochran, Adv. Phys. 42, 5 (1993).

${ }^{15}$ For small deviations from the the equilibrium profile $\left(\left\{\theta_{n}^{0}\right\}\right)$ the energy has the form $E\left(\left\{\theta_{n}^{0}+\delta \theta_{n}\right\}\right)=E\left(\left\{\theta_{n}^{0}\right\}\right)+Q$, where $Q$ $=\frac{1}{2} \Sigma_{i, j} m_{i j} \delta \theta_{i} \delta \theta_{j}$ is a positive definite quadratic form.

${ }^{16}$ W. Saslow and D. L. Mills, Phys. Rev. 171, 488 (1968). 